Please do not remove this page

RMIT

UNIVERSITY

\title{
Reversible resistive switching behaviour in CVD grown, large area MoOx
}

rahman, Fahmida; Ahmed, Taimur; Walia, Sumeet; Mayes, Edwin; Sriram, Sharath; Bhaskaran, Madhu; Balendhran, Shiva

https://researchrepository.rmit.edu.au/esploro/outputs/9921863802301341/filesAndLinks?institution=61RMIT_INST\&index=null

rahman, F., Ahmed, T., Walia, S., Mayes, E., Sriram, S., Bhaskaran, M., \& Balendhran, S. (2018). Reversible resistive switching behaviour in CVD grown, large area MoOx. Nanoscale, 10(42), 19711-19719.

https://doi.org/10.1039/C8NR04407D

Document Version: Accepted Manuscript

Published Version: https://doi.org/10.1039/C8NR04407D

Repository homepage: https://researchrepository.rmit.edu.au

(C) 2018 The Royal Society of Chemistry

Downloaded On 2023/04/26 23:18:39 +1000

Please do not remove this page 
Thank you for downloading this document from the RMIT Research Repository.

The RMIT Research Repository is an open access database showcasing the research outputs of RMIT University researchers.

RMIT Research Repository: http://researchbank.rmit.edu.au/

\section{Citation:}

Rahman, F, Ahmed, T, Walia, S, Mayes, E, Sriram, S, Bhaskaran, M and Balendhran, S 2018, 'Reversible resistive switching behaviour in CVD grown, large area MoOx', Nanoscale, vol. 10, no. 42, pp. 19711-19719.

See this record in the RMIT Research Repository at:

https://researchbank.rmit.edu.au/view/rmit:50207

Version: Accepted Manuscript

Copyright Statement:

(C) 2018 The Royal Society of Chemistry

\section{Link to Published Version:}

https://researchbank.rmit.edu.au/view/rmit:50207 


\title{
Journal Name
}

\section{Reversible resistive switching behaviour in layered $\mathrm{MoO}_{x}$}

Received 00th January 20xx, Accepted 00th January 20xx

DOI: $10.1039 / x 0 x x 00000 x$

\author{
Fahmida Rahman ${ }^{1 *}$, Taimur Ahmed ${ }^{1}$, Sumeet Walia ${ }^{1}$, Edwin Mayes ${ }^{2}$, Sharath Sriram ${ }^{1}$, Madhu \\ Bhaskaran ${ }^{1}$ and Sivacarendran Balendhran ${ }^{1^{*}}$
}

\begin{abstract}
Non-volatile resistive memory devices are theorized to be the most promising pathway, towards analog memories and neuromorphic computing. Two-dimensional $\mathrm{MoO}_{3}$ is a versatile planar transition metal oxide, whose properties can be readily tuned, rendering it anywhere from a wide bandgap semiconductor to semi-metallic. Successful integration of such a planar metal oxide into resistive memories, can enable adaptive and low power memory applications. Here, we investigate the non-volatile and reversible resistive switching behaviour of oxygen deficient $\mathrm{MoO}_{x}$ in a cross-point metal/insulator/metal (MIM) architecture. Layered $\mathrm{MoO}_{x}$ films are synthesised using chemical vapour deposition (CVD) and reveal excellent resistive switching performance with relatively low electroforming and operating voltages. Switching ratios of $\sim 10^{3}$ and stable data retention of $>10^{4} \mathrm{~s}$ are achieved. As such, this work demonstrates the viability of $\mathrm{MoO}_{x}$, as a resistive memory element and paves the way for future two-dimensional resistive memory technologies.
\end{abstract}

\section{Introduction}

In the current digital era, next generation smart devices demand high-speed, efficient and high-density electronics. Existing silicon-based technology has reached its theoretical and physical limitations. It becomes notably difficult to reliably retain charge in the existing Si-based technology, as the modern device structure shrinking by the day. ${ }^{1}$ Resistive memory devices or memristors, are two-terminal 'memory resistors' that can store information in the form of resistance states. The resistive states of such a device rely on the history of applied voltage and can be reversibly switched. ${ }^{2}$ In theory, non-volatile resistive memory technology holds potential, ${ }^{3-5}$ as it possesses superior features; including non-volatile electrical switching, longer memory retention, high data density and compatibility with contemporary complementary metal oxide semiconductor (CMOS) technology. ${ }^{6-8}$ They can also be easily scaled down to less than $10 \mathrm{~nm}, 6$ and offer ease of fabrication. ${ }^{9}$ Several metal oxides such as, $\mathrm{TiO}_{2},{ }^{10-12} \mathrm{WO}_{3},{ }^{13}$ $\mathrm{VO}_{2},{ }^{14} \mathrm{STO},{ }^{15-18} \mathrm{ZnO}^{19,} 20$ have been used as the active material, which exhibit reversible resistive switching behaviours. However, as the field is still at its infancy, significant progress is yet to be made, in order to integrate memristor technology into the existing CMOS based nano-electronics.

Molybdenum oxide $\left(\mathrm{MoO}_{x}\right)$ is a potential two-dimensional transition metal oxide with enhanced electronic transport properties, which can be easily tuned via the manipulation of

\footnotetext{
${ }^{1}$ Functional Materials and Microsystem Research Group and Micro Nano Research Facility, RMIT University, Melbourne, Victoria 3000 (Australia).

${ }^{2}$ RMIT Microscopy and Microanalysis Facility, RMIT University, Melbourne,

Victoria 3000 (Australia).

Email: fahmida.rahman@gmail.com;shiva.balendhran@rmit.edu.au
}

its stoichiometry. ${ }^{21,} 22 \mathrm{MoO}_{x}$ can be obtained in two stable states, $\mathrm{MoO}_{2}$ and $\mathrm{MoO}_{3}$. $\mathrm{MoO}_{2}$ is the lowest oxidation state $\mathrm{Mo}^{4+}$, which exists as a distorted rutile-type monoclinic structure and shows semi-metallic properties. On the other hand, the highest oxidation state of $\mathrm{MoO}_{3}$ is a wide bandgap semiconductor that occurs in an orthorhombic-layered structure. In-between exists the oxygen-deficient intermediate state $\mathrm{MoO}_{3-x}$, that can be tuned to behave anywhere from semi-metallic to insulating. To date resistive memory behaviour of bulk $\mathrm{MoO}_{3}$ and composites have been studied. ${ }^{23}$ ${ }^{26}$ However, exploiting the planar form of $\mathrm{MoO}_{x}$ in its multioxidation states has the potential to offer the desirable stoichiometries to realize faster switching, and low power resistive memory elements.

In this work, CVD grown thin layers ( $25 \mathrm{~nm}$ ) of $\mathrm{MoO}_{x}$ has been introduced as a resistive switching oxide for non-volatile memory applications, in cross-planar device architecture. Extensive characterisation has been carried out on the asgrown material to establish the chemical composition and crystalline structure. The suitability of the material in nonvolatile memory applications has been experimentally assessed via cyclic repeatability and memory retention tests.

\section{Experimental}

\section{CVD growth of $\mathrm{MoO}_{x}$}

$\mathrm{MoO}_{x}$ film with thickness of 25-30 nm was deposited on patterned on bottom $\mathrm{Pt} / \mathrm{Ti}$ electrodes on $\mathrm{SiO}_{2} / \mathrm{Si}$ substrates using chemical vapour deposition at $530{ }^{\circ} \mathrm{C}$. The precursor $\left(\mathrm{MoO}_{3}\right.$ powder, $\left.100 \mathrm{mg}\right)$ was placed in the middle zone $\left(785^{\circ} \mathrm{C}\right)$ of a horizontal 3-zone furnace. The growth substrates were placed downstream in the third zone, at $530{ }^{\circ} \mathrm{C}$. Argon 
was used as the carrier gas at a rate of $100 \mathrm{sccm}$ and a chamber pressure of $300 \mathrm{mT}$ has been maintained.

\section{Material characterisation}

The thickness of $\mathrm{MoO}_{x}$ layer was measured using Dimension Icon Bruker atomic force microscope under ScanAsyst Air mode. LabRam HR Evolution Raman Spectrometer (laser excitation of $532 \mathrm{~nm}$ with a $50 \times$ objective) has been utilised to assess the composition of the as-grown $\mathrm{MoO}_{x}$, prior to device fabrication. X-ray diffractograms were collected using a Bruker D4 Endeavor XRD instrument with monochromatic $\mathrm{Cu} \mathrm{K \alpha}$ as radiation source, within an angular range $2 \theta$ from $20^{\circ}$ to $60^{\circ}$. $\mathrm{X}$-ray photoelectron spectroscopy was performed using a Thermo Scientific K-alpha X-ray Photoelectron Spectrometer with an $\mathrm{Al} \mathrm{K} \alpha$ radiation source with energy of $1485 \mathrm{eV}$. Adventitious carbon peak at $284.8 \mathrm{eV}$ has been used as the calibration reference for fitting of the core level spectra of the principal elements. A FEI Nova NanoSEM has been utilised to characterise the morphology of the devices after patterning top electrode.

TEM lamella samples have been prepared using focused ion beam milling in a FEI Scios DualbeamTM scanning electron microscope. Cross-sectional analysis and energy-dispersive $X$ ray spectroscopy are performed on a JEOL $2100 \mathrm{~F}$ scanning transmission electron microscope (STEM) with attached Tridium Gatan image filter.

\section{Cross-point device fabrication}

$\mathrm{MoO}_{x}$ memory devices in cross-point configuration (square overlap areas with sides of 20-100 $\mu \mathrm{m}$ ) were fabricated on $\mathrm{SiO}_{2} / \mathrm{Si}$ substrates, using a three-step photolithography and lift-off process. Metal thin films of $30 \mathrm{~nm}$ Pt with a $5 \mathrm{~nm} \mathrm{Ti}$ adhesion layer were deposited as bottom electrodes via electron beam evaporation at room temperature. Subsequently, $\mathrm{MoO}_{\mathrm{x}}$ was deposited over the bottom electrodes using a CVD process. Thereafter, $30 \mathrm{~nm}$ Pt (with a $10 \mathrm{~nm} \mathrm{Ti}$ adhesion layer) was deposited via electron beam evaporation. Finally, photolithography and selective $\mathrm{MoO}_{x}$ etching was utilised to reveal the bottom electrode pads, for electrical characterisation.

\section{Electrical Characterisation}

Two-terminal electrical characterisation was performed using an Agilent B2912A source with a micromanipulator probe station. Bipolar current-voltage (I-V) sweep cycles, memory retention and cyclic endurance tests were performed on the fabricated devices. All electrical characterisations were performed by applying a bias voltage to the bottom electrode with the top electrode being grounded.
Atomic force microscopy (AFM) has been utilized to measure the thickness of the CVD grown oxide films. Fig. 1a shows the AFM image and the thickness profile of the as-deposited material. The thickness profile revealed that the as-grown material is $\sim 25 \mathrm{~nm}$ thick (Fig. 1a). Raman spectroscopy has been employed to assess the structural composition of the asgrown oxide layer. Fig. $1 \mathrm{~b}$ shows the micro-Raman spectrum of the CVD grown film, in which Raman signature peaks corresponding to $\mathrm{MoO}_{x}\left(\mathrm{MoO}_{2}, \mathrm{MoO}_{3-\mathrm{x}}\right.$ and $\left.\mathrm{MoO}_{3}\right)$ were identified. The low intensity characteristic peaks at $356 \mathrm{~cm}^{-1}$ and $732 \mathrm{~cm}^{-1}$ confirm the presence of an intermediate oxidation state $\left(\mathrm{MoO}_{3-\mathrm{x}}\right)$ indicative of localized $\mathrm{Mo}^{5+}$, produced due to oxygen vacancies. ${ }^{27,} 28$ The high intensity peak at $201 \mathrm{~cm}^{-1}$ and relatively lower intensity peaks at $493 \mathrm{~cm}^{-1}$ and $567 \mathrm{~cm}^{-1}$ suggest the presence of $\mathrm{Mo}^{4+}$ in the as-grown material. ${ }^{27,29}$ Peaks observed at $820 \mathrm{~cm}^{-1}$ and $995 \mathrm{~cm}^{-1}$ can be correlated to the $\mathrm{Mo}^{6+}$ oxidation state of $\alpha-\mathrm{MoO}_{3} .{ }^{27,} 30$ The Raman analysis indicates that a mixture of molybdenum oxide species $\left(\mathrm{Mo}^{4+}, \mathrm{Mo}^{5+}\right.$, and $\left.\mathrm{Mo}^{6+}\right)$ are present in the as-deposited film.

To further analyse the compositional ratio of the oxide species, high resolution X-ray photoemission spectroscopy (XPS) measurements were carried out (Fig. 1c). Adventitious carbon C 1 s peak at $284.8 \mathrm{eV}$ has been taken as a reference which shows no charge shifting. ${ }^{31}$ The presence of $C 1$ s peak is due to the expected carbon contamination from the environment. Typically, a Mo 3d-doublet with a spin-orbit component of Mo $3 d_{5 / 2}$ and $M o 3 d_{3 / 2}$ is associated with a single oxidation state in molybdenum oxides. The de-convolution and the curve fitting of the XPS spectrum reveals that Mo 3d spectrum can be well fitted with three sets of $3 d$ doublets in the form of a Gaussian function, which are corresponding to three oxidation states of Mo. The peak fitting has been done with a fixed energy gap of $3.2 \mathrm{eV}$ for the doublet of spin-orbit coupling peaks. The convoluted peaks also show that the ratio of the integral areas between two doublets is $3: 2$ and have the same full width at half maximum (FWHM) values. ${ }^{30,32}$ The binding energy values corresponding to $232.7 \mathrm{eV}$ (Mo 3 $\mathrm{d}_{5 / 2}$ ) and $235.9 \mathrm{eV}\left(\mathrm{Mo} \mathrm{3} \mathrm{d}_{3 / 2}\right.$ ) are characteristics of oxidation state of $\mathrm{Mo}^{6+} .33,34$ Whereas,

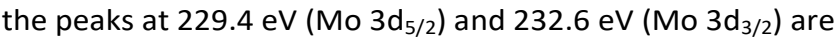
assigned to $\mathrm{Mo}^{4+}$ oxidation state. ${ }^{35} \mathrm{In}$ addition, a third pair of doublets at $231.3 \mathrm{eV}$ (Mo 3d $\mathrm{d}_{5 / 2}$ ) and $234.5 \mathrm{eV}$ (Mo 3d $\mathrm{d}_{3 / 2}$ ) corresponding to $\mathrm{Mo}^{5+}$ are also observed. ${ }^{36}$ Based on the XPS analysis, it can be concluded that the as-deposited material is a combination of multiple oxidation states of $\mathrm{Mo}\left(26 \% \mathrm{Mo}^{4+}\right.$, $46 \% \mathrm{Mo}^{5+}$ and $\left.28 \% \mathrm{Mo}^{6+}\right)$. The calculated atomic mass percentages of each oxidation state and their corresponding binding energy values are listed in Table 1.

\section{Results and discussion}


Table 1 Binding energy values for Mo $3 \mathrm{~d}$ spin-orbit component of molybdenum oxide at various oxidation states

\begin{tabular}{|c|c|c|c|}
\hline \multirow{2}{*}{$\begin{array}{c}\text { Oxidation } \\
\text { state }\end{array}$} & \multicolumn{2}{|c|}{ Binding Energy value $(\mathrm{eV})$} & \multirow{2}{*}{$\begin{array}{c}\text { Concentration } \\
\text { (Atomic\%) }\end{array}$} \\
\cline { 2 - 3 } $\mathrm{Mo}^{4+}$ & 229.4 & 232.6 & $26 \%$ \\
\hline $\mathrm{Mo}^{5+}$ & 231.3 & 234.4 & $46 \%$ \\
\hline $\mathrm{Mo}^{6+}$ & 232.7 & 235.9 & $28 \%$ \\
\hline
\end{tabular}

To investigate the crystallinity of the as-grown material, X-ray diffraction (XRD) patterns were recorded (Fig. 1d). The diffractogram shows two dominant peaks at $26.1^{\circ}$ and $37.5^{\circ}$, corresponding to (011) and (002) planes, respectively, of monoclinic $\mathrm{MoO}_{2}$ (JCPDS No. 78-1070). ${ }^{37,} 38$ There are two comparatively low intensity peaks at $38.9^{\circ}$ and $40.6^{\circ}$ correspond to (060) plane of $\mathrm{MoO}_{3}$ (JCPDS No. 05-0508) and (203) plane of $\mathrm{MoO}_{3-x}$ (JCPDS No. 05-0337), respectively. The XRD patterns indicate a dominant presence of monoclinic $\mathrm{MoO}_{2}$ co-existing with $\mathrm{MoO}_{3-x}$ and $\mathrm{MoO}_{3}$, further validating the conclusions derived from the Raman and XPS analyses. However, once the material is subject to electric fields (an electroforming process), it transforms to predominantly $\mathrm{MoO}_{3}$ $x$ (discussed later). (a)

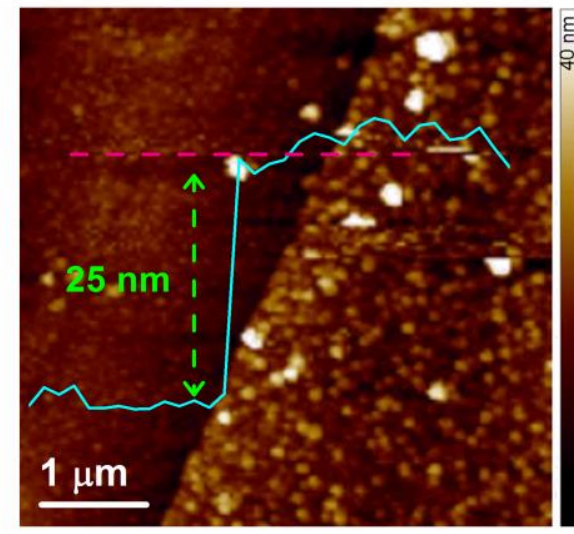

(c)

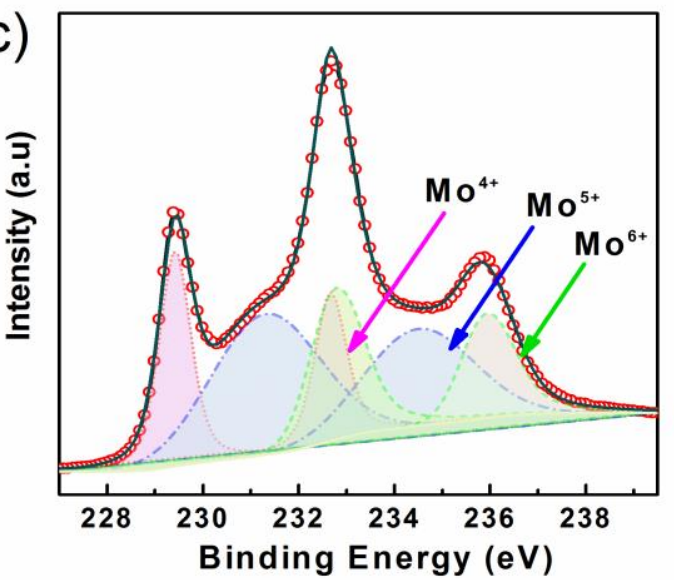

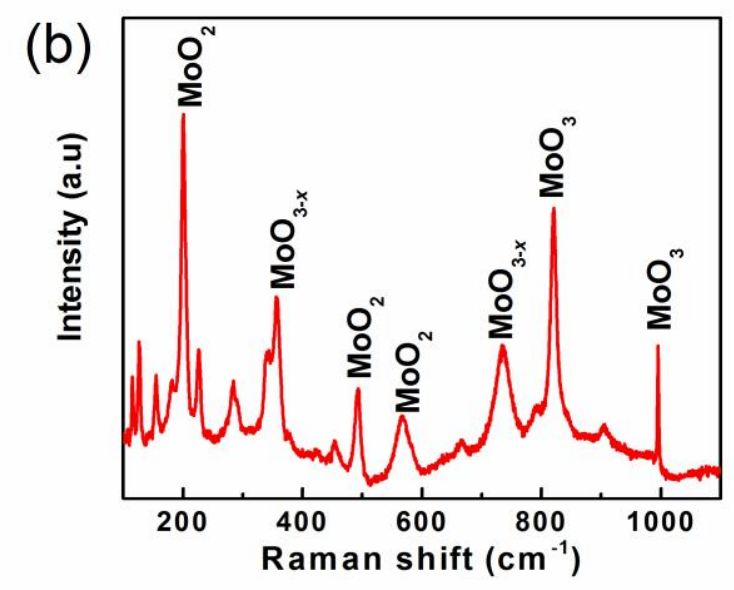

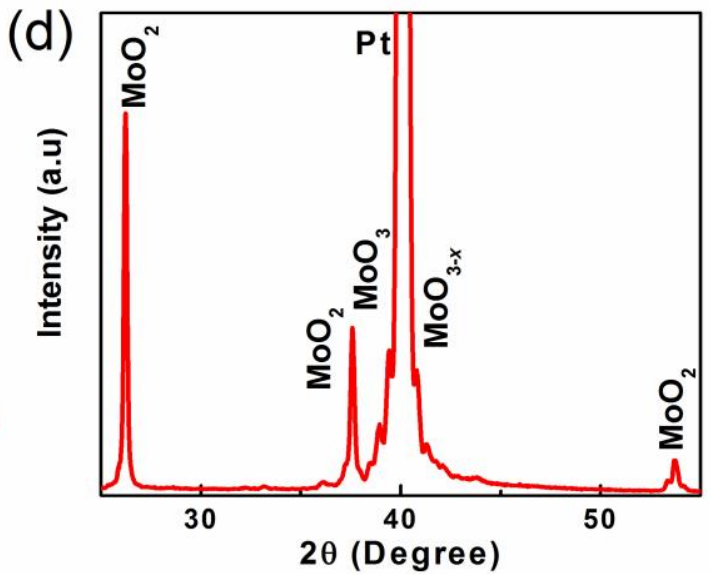

Figure 1 Characterisation of chemical vapour deposition grown $\mathrm{MoO}_{x}$ : (a) Atomic force micrograph and thickness measurement, (b) micro-Raman spectra, (c) X-ray photoelectron spectra of Mo 3d XPS spectra with fitted spin-orbit components, and (d) X-ray diffractogram.

Typically, resistive states of the memristors are electrically manipulated by controlling conductive filaments that are composed of oxygen vacancies. Since the oxygen vacancies are widely dispersed in metal oxides, an application of a large electric field across the material is required for the initial formation of conductive filaments (electroforming process). Upon electroforming, the material switches from a high resistive state (HRS) to a low resistive state (LRS).

Subsequently, depending on the polarity of the applied electric field, the oxygen vacancies can be scattered to rupture the 
conductive paths (to achieve HRS) or concentrated to re-form conductive filaments (to achieve LRS). ${ }^{10,15,39-41}$ Owing to the formation and rupture of the conductive filaments, a reversible behaviour in the resistive state of the material can be observed. The current-voltage $(I-V)$ characteristics curve of such a device exhibits a pinched hysteresis trend, illustrating the reversible switching between the low and high resistive states of the material.

Cross-point memory cells were fabricated on large area CVD grown $\mathrm{MoO}_{x}$ (see experimental section). Figure 2(a) shows a schematic illustration of the $\mathrm{Pt} / \mathrm{Ti} / \mathrm{MoO}_{x} / \mathrm{Pt} / \mathrm{Ti}$ cross-point memory cell while figure 2 (b) and (c), present optical and scanning electron micrographs of an as fabricated device, respectively. To electroform the as-fabricated devices, a set of positive bias ( 0 to $+5 \mathrm{~V}$ ) and subsequent negative bias was applied across the bottom and top electrodes. A current compliance limit of $800 \mu \mathrm{A}$ was set to prevent any dielectric breakdown of the device. The electroforming process was observed to take place at an applied voltage of $-2.7 \mathrm{~V}$, where the device resistance was reduced by several orders of magnitude.
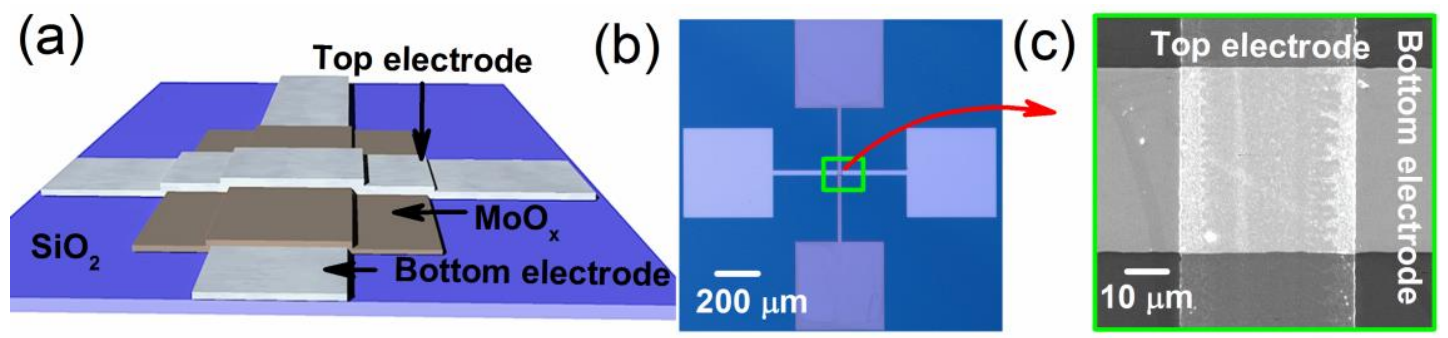

Figure 2 Schematic illustration of the cross-point memristor device architecture. (b) Optical and (c) scanning electron micrographs of an as-fabricated memristor.

Following the electroforming process, several cycles of bipolar switching voltage were applied, and the devices show reversible resistive switching behaviour over multiple cycles. Fig. 3a shows the electrical characteristics of an electroformed memory cell, when subjected to voltage sweeps of $-2.0 \mathrm{~V}$ to $+2.5 \mathrm{~V}$. Minor fluctuations in threshold voltages are observed in the pinched hysteresis loops. Such negligible fluctuations are attributed to the variations in /HRS, which is caused by the existence of conductive filamentary paths that can survive the RESET process, and the stochastic nature of filament formation. ${ }^{42}$ Fig. $3 b$ shows the statistical distribution of the switching voltages ( $V_{\text {SET }}$ and $V_{\text {RESET }}$ ), observed in the cyclic switching results presented in Fig $3 a$. The $V_{\text {SET }}$ ranges from -0.7 to $-1.3 \mathrm{~V}$ with a mean value of $-1.09 \mathrm{~V}$ and the $V_{\text {RESET }}$ ranges from +2.2 to $+2.6 \mathrm{~V}$ with a mean value of $+2.3 \mathrm{~V}$. The stability of the resistive states was measured over a long period of time, to acquire the memory retention characteristics of the electroformed devices. Fig. 3c shows the stability of the resistive states for durations of over $10^{4} \mathrm{~s}$, at room temperature. Here, the ON/OFF ratio was consistently maintained above $10^{3}$ without any deterioration, at a read voltage of $500 \mathrm{mV}$. As such the retention test reveals robust non-volatile behaviour of the devices and reliability of the material for memory applications. Electrical endurance tests were performed at room temperature to evaluate the cycle-tocycle stability and reproducibility of $\mathrm{MoO}_{x}$-based devices. The current values ( LRS $_{\text {LR }}$ and $I_{\text {HRS }}$ ) of a memory cell were extracted at a read voltage of $500 \mathrm{mV}$, while the device was subjected to alternating write and erase cycles (Fig. 3d). The device was observed to have successfully retained the two distinct resistance states for over 100 repetitive cycles while maintaining a switching ratio of $\sim 10^{3}$. Although, the resistance values in LRS are mostly uniform, the resistance values in HRS are dispersive. Such behaviour suggests that several residual filamentary paths may exist in the HRS. ${ }^{42}$ However, the cyclic endurance of the device seems to suffer beyond 100 cycles, resulting in a dramatic reduction of the switching ratio. Endurance failure in such devices is caused by Joule heating and repetitive redox reactions leading to the formation of permanent filamentary paths, at which point the devices continue to remain in their LRS. 


\section{Journal Name}

\section{ARTICLE}

(a)

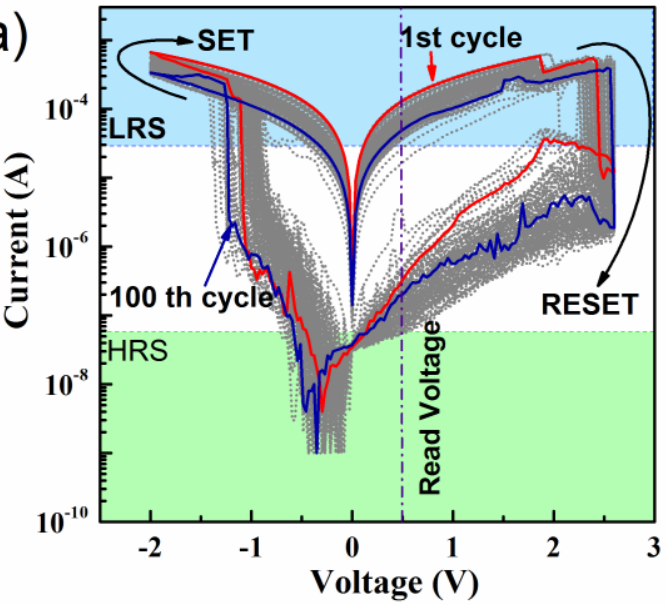

(c)

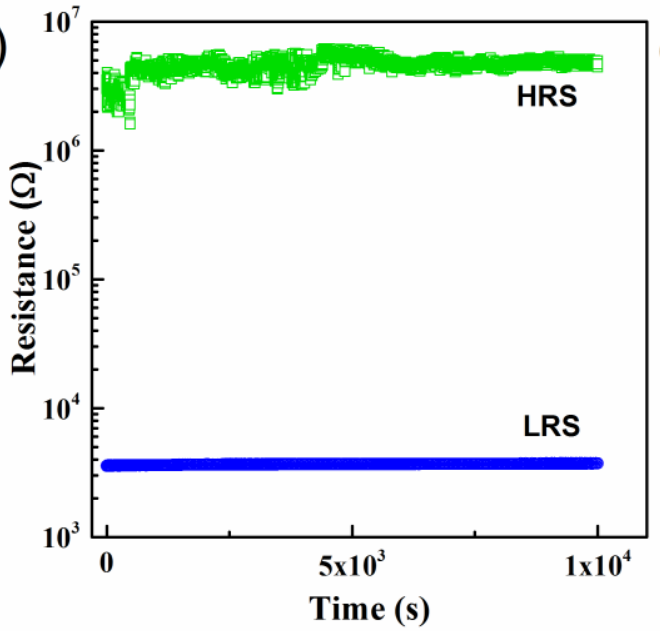

(b)

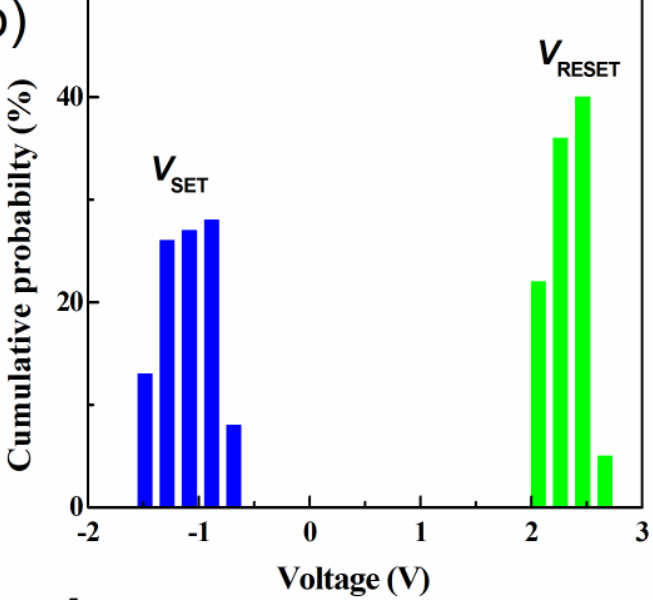

(d)

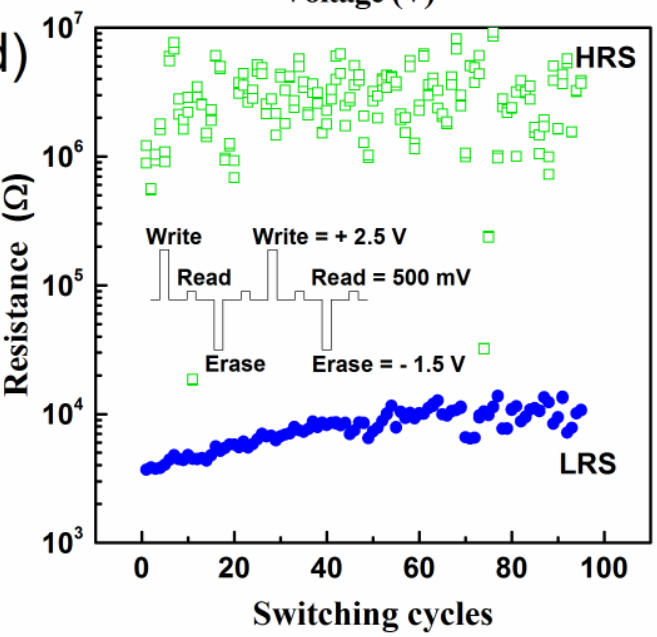

Figure 3 (a) Characteristic current-voltage curves of a memory cell subjected to 100 cycles of switching. (b) Statistical analysis of the switching voltages over 100 cycles of repetitive switching. (c) Retention of the resistive state with respect to time. (d) Performance endurance with respect to number of repetitive cycles. (Resistance values obtained at a read voltage of $500 \mathrm{mV}$ ).

To understand the charge transfer and switching mechanism in the $\mathrm{MoO}_{x}$ memory clearly, $I-V$ characteristics curves are plotted on a double logarithmic scale. Figure 4(a), (b) show the $\mathrm{I}-\mathrm{V}$ curves for both positive and negative biasing. The characteristics curve shows Ohmic conduction behaviour $(/ \infty V)^{43,44}$ in LRS region for both positive and negative biasing. The $I-V$ characteristics curve in HRS during positive biasing, shows $10 \mathrm{~V}$ relationship at lower voltages and $1 \infty \mathrm{V} \geq 2$ at higher voltages, indicating a space charge limited conduction (SCLC) mechanism. ${ }^{45}$ The HRS region during negative biasing can be divided in to two parts. In the lower voltage region, the current decreases with an increase in applied voltage, which suggests the existence of a reverse built-in electric field. ${ }^{46-48}$ This is attributed to the interfacial Ti layer, which has a tendency to oxidise, creating a reverse built in potential near the top electrode interface. Once this voltage is breached, the current then increases with an increasing bias voltage, which is in good agreement with SCLC mechanism. 
Based on the above results, a physical model of oxygen vacancy migration and filaments formation has been developed and a schematic illustration of the resistive switching mechanism is presented in Figure 4(c). The pristine $\mathrm{MoO}_{x}$ cells contain randomly distributed positively charged oxygen vacancies (the presence of $\left.\mathrm{Mo}^{5+}\right),{ }^{49}$ which act as mobile donors in the oxide layers. ${ }^{50}$ As the applied voltage increases, the oxygen vacancies present in the oxide layers migrate in the direction of applied field and accumulate near the bottom electrode. Eventually this oxygen deficient region forms single/multiple conductive filaments between the electrodes, leading to LRS of the material. Subsequently, a positive bias at the bottom electrode $(\mathrm{Pt})$ repels the oxygen vacancies from the electrode, resulting in the partial rupture of the conductive filaments. Consequently, the memory cell reverts back to a HRS between the electrodes (RESET). Conversely, a smaller negative bias (relative to the electroforming voltage), enables the reformation of the filaments and allow the cell to switch back to a LRS (SET). As such, the partial rupture and the reformation of filaments composed of oxygen vacancies, result in the resistive switching of layered $\mathrm{MoO}_{x}$ (a)

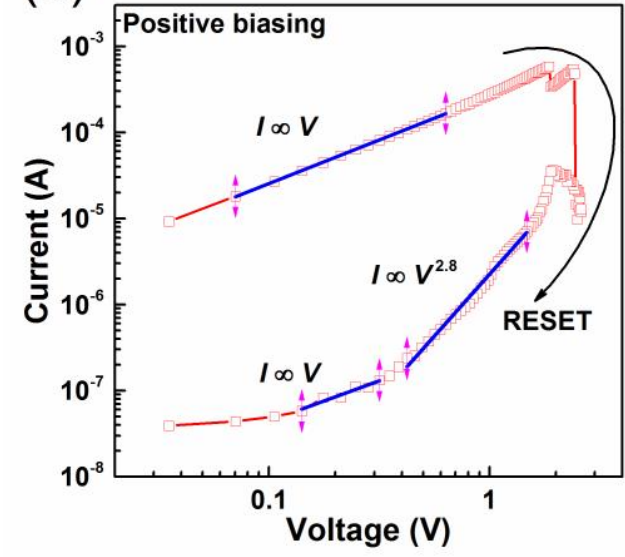

(b)

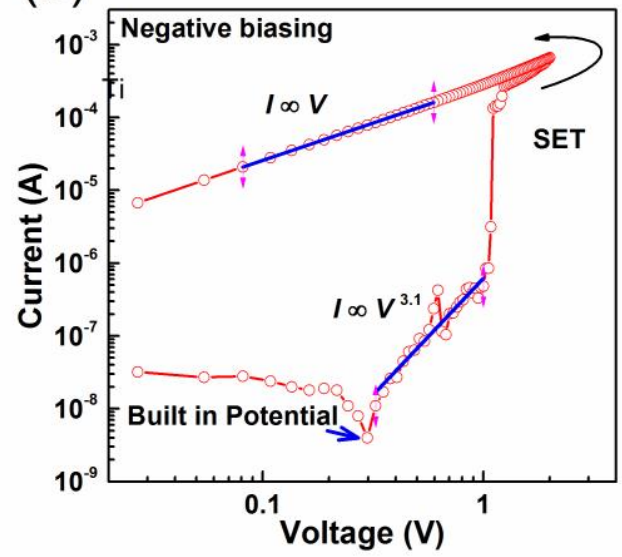

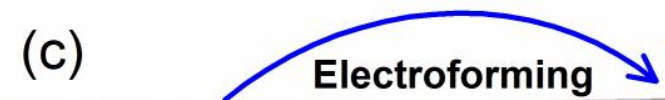
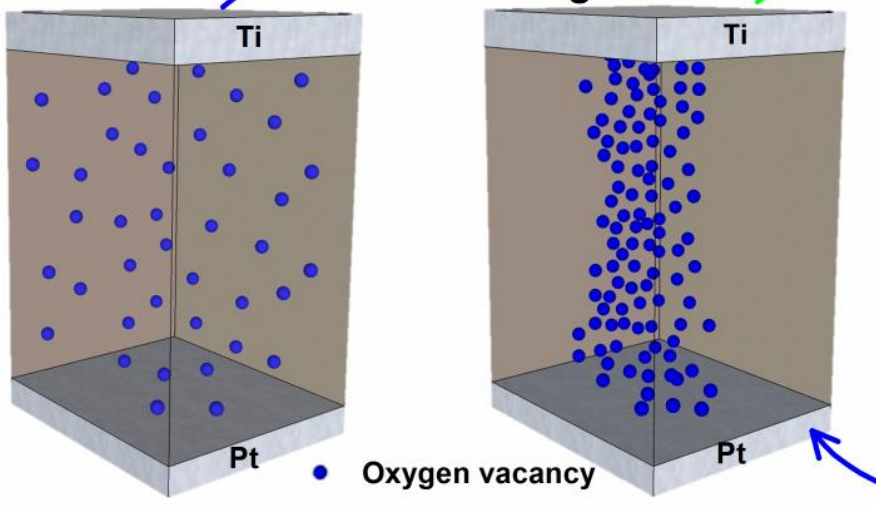

\section{RESET}

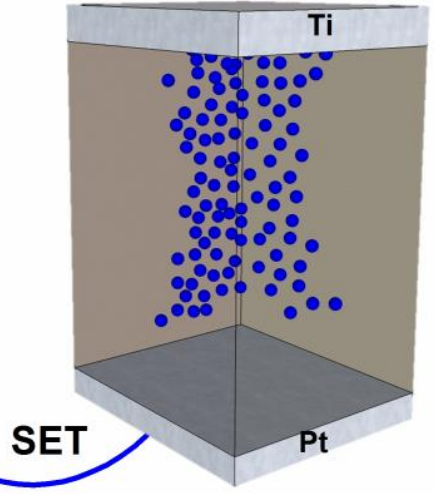

Figure $4 \mathrm{I}-\mathrm{V}$ characteristics curves of both (a) positive and (b) negative bias regions of the $\mathrm{MoO}_{x}$ memory device plotted in a double-logarithmic scale. Both LRS and HRS are included on this plot. (c) Schematic illustration of electroforming and subsequent switching mechanisms in $\mathrm{MoO}_{x}$ memory cells. Bottom electrode is biased and the top electrode remains grounded.

To analyse the structural integrity of the resistive memory cells, cross-sectional high-resolution transmission electron microscopy (HRTEM) of pristine and electroformed memory cells was undertaken (Fig. 5). The MIM interface can be clearly identified on the HRTEM images for both the pristine and electroformed devices. The HRTEM image of a pristine memory cell reveals the presence of both polycrystalline and amorphous phases in the $\mathrm{MoO}_{x}$ stack (inset of Fig. 5a). For the compositional analysis of the distinct phases, three regions of interest (ROIs) were selected (highlighted in Fig. 5a). The fast Fourier transform (FFT) diffraction pattern of the first ROI shows the amorphous nature of the film. In the other two distinct ROIs, (001) plane of $\alpha-\mathrm{MoO}_{3}$ and (002) plane of $\mathrm{MoO}_{2}$ were identified. This agrees with the XPS characterisations confirming the existence of multiple oxidation states in the metal oxide layer $\left(\mathrm{MoO}_{3}, \mathrm{MoO}_{3-x}\right.$, and $\left.\mathrm{MoO}_{2}\right)$. However, the cross-sectional HRTEM image of the electroformed device reveals only one type of crystalline structure, corresponding to 
$\mathrm{MoO}_{3} / \mathrm{MoO}_{3-x}$ (Fig. 5b). Inset at the top shows the FFT diffraction pattern of the selected ROI. The diffraction spots with d-spacing $0.36 \mathrm{~nm}$ are indexed to (001) plane corresponding to orthorhombic $\mathrm{MoO}_{3} / \mathrm{MoO}_{3-x}$. The phase change of the material suggests that the process of electroforming predominantly oxidises the $\mathrm{Mo}^{4+}$ to $\mathrm{Mo}^{5+} / \mathrm{Mo}^{6+}$ states. The diffraction patterns corresponding to (001) are used to generate the inverse FFT, which highlights the dominant existence of $\mathrm{MoO}_{3} / \mathrm{MoO}_{3-x}$, in the ROI (Fig. 5b, bottom right inset). Such a transition is expected, as the lower oxidation states $\left(\mathrm{Mo}^{4+}\right)$ get oxidised into higher states $\left(\mathrm{Mo}^{5+} / \mathrm{Mo}^{6+}\right)$, due to Joule heating induced by the localized electrical stress. Based on the TEM analyses, it can be conferred that the applied electric field, changes the material composition from its multi-oxidation state to a substoichiometric form of $\mathrm{MoO}_{3}$.

To ensure the integrity of the MIM architecture post electroforming, an elemental analysis on the cross section of the devices was carried out using energy dispersive spectroscopy (EDS). Cross-sectional scanning transmission electron microscopy (STEM) image and the corresponding elemental EDS maps carried out on a pristine (Fig. 5c) and an electroformed device (Fig. $5 \mathrm{~d}$ ) are presented. The MIM structure was well maintained pre- and post-electroforming process, with no diffusion of metal observed through the oxide $\left(\mathrm{Pt} / \mathrm{Ti} / \mathrm{MoO}_{x} / \mathrm{Pt} / \mathrm{Ti}\right)$. Slight overlap of the elements in the interfacial region is attributed to the localised Joule heating and the resolution of the imaging technique. It is inaccurate to draw a conclusion from the oxygen map, as the cross-sectional sample preparation involves high energy focussed ion beam milling process which typically results in oxygen contamination. Based on these results, metal diffusion is eliminated as a contributing factor in the switching process and the $\mathrm{MoO}_{x}$ layer is concluded as the sole contributor to the observed resistive switching. (a)

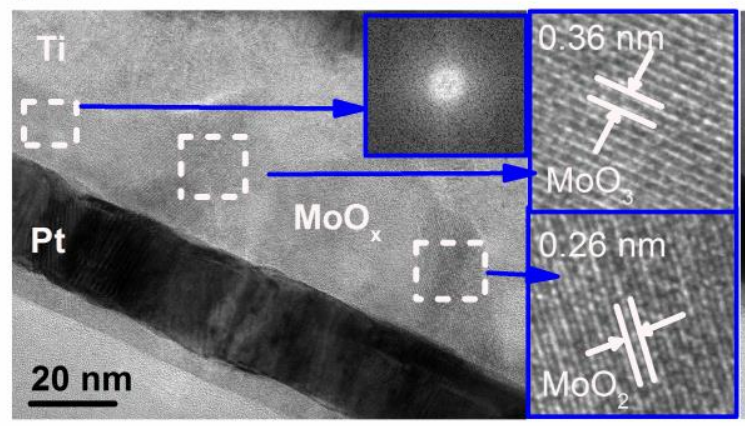

(b)

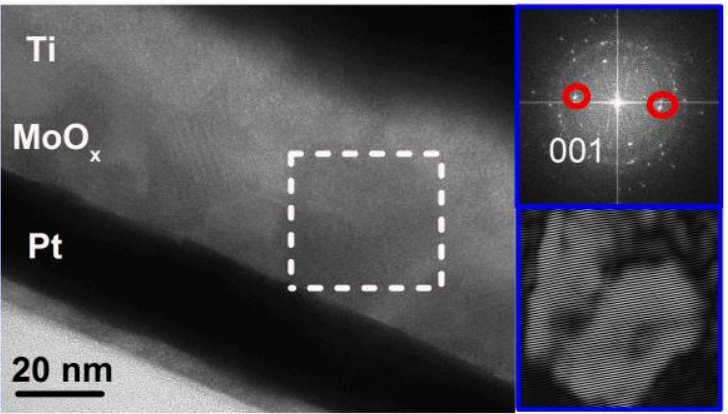

\section{(c)}
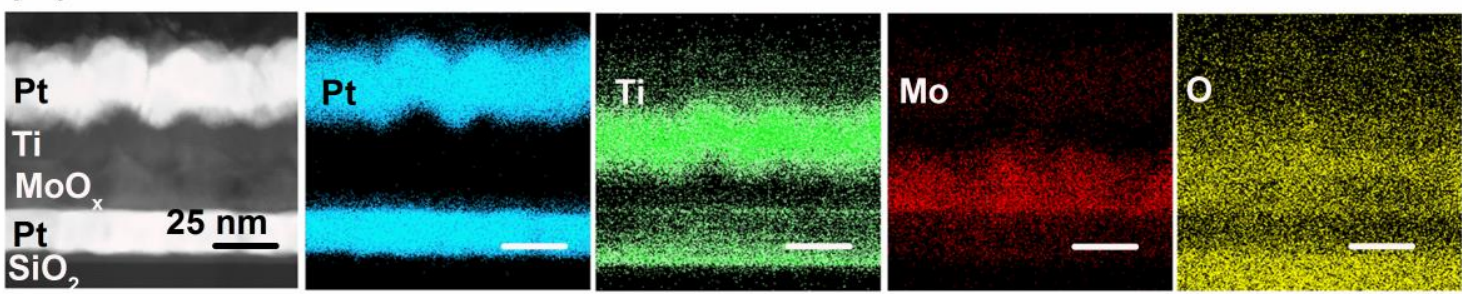

\section{(d)}

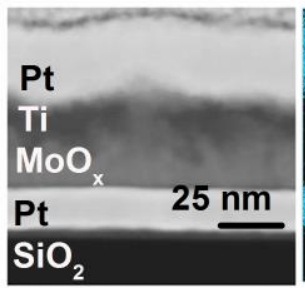

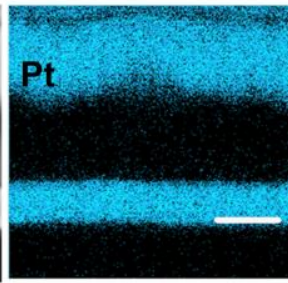
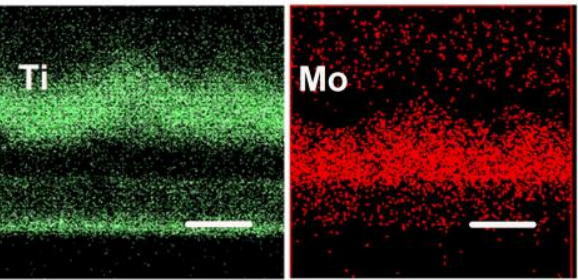

Figure 5 Structural analyses of a pristine and an electroformed device. (a) The cross-sectional HRTEM image of a pristine cell. Insets showing different lattice parameters indicating the co-existence of multi-oxidation state of molybdenum oxide (MoO2 and MoO3). (b) HRTEM image of an electroformed cell. Insets are the FFT image and inverse FFT of the ROI in (b). The cross-sectional EDS elemental maps of (c) a pristine device cell and (d) an electroformed cell. 


\section{Journal Name}

\section{Conclusions}

In conclusion, resistive switching behaviour has been demonstrated in CVD grown ultra-thin $\mathrm{MoO}_{x}$ films. The switching performance of cross-point $\mathrm{MoO}_{x}$ memory devices are driven by the presence of oxygen vacancies, due to the various oxidation states co-existing in the as-grown material. The composition of the as-grown $\mathrm{MoO}_{x}$ films are thoroughly characterised through multiple spectroscopic techniques. However, it is found that post electroforming, the material composition changes to an oxygen-deficient $\mathrm{MoO}_{3-x}$ film. The room temperature memory retention tests show high ON/OFF ratios exceeding $\sim 10^{3}$ for durations over $10^{4} \mathrm{~s}$. The cyclic endurance of the memory devices shows that they remain stable over 100 cycles while maintaining a switching ratio of $\sim 10^{3}$. As such, this work showcases the adaptability of $\mathrm{MoO}_{x}$ nanofilms, in non-volatile resistive memory applications, as a precursor for fully integrated two-dimensional film electronics.

\section{Conflicts of interest}

There are no conflicts to declare.

\section{Acknowledgements}

The authors acknowledge support from the Australian Research Council (ARC) with project and personnel support through DE150100909 (SB), DE160100023 (MB), and DP140100170 (MB) and equipment funding through LE110100223 and LE150100001. The authors also acknowledge the facilities and technical assistance of the Micro Nano Research Facility and RMIT Microscopy and Microanalysis Facility.

\section{References}

1. A. I. Kingon, J.-P. Maria and S. K. Streiffer, Nature, 2000, 406, 1032-1038.

2. R. Waser, R. Dittmann, G. Staikov and K. Szot, Adv. Mater., 2009, 21, 2632-2663.

3. A. Sawa, Mater. Today, 2008, 11, 28-36.

4. K. M. Kim, D. S. Jeong and C. S. Hwang, Nanotechnology, 2011, 22, 254002.

5. F. Yoshihisa, Jpn. J. Appl. Phys., 2013, 52, 040001.

6. B. Mohammad, M. A. Jaoude, V. Kumar, D. M. Al Homouz, H. A. Nahla, M. Al-Qutayri and N. Christoforou, Nanotechnol. Rev., 2016, 5.

7. V. K. Sangwan, D. Jariwala, I. S. Kim, K. S. Chen, T. J. Marks, L. J. Lauhon and M. C. Hersam, Nat. Nanotechnol., 2015, 10, 403-406.
2. Liang, K. Li, C. Xiao, S. Fan, J. Liu, W. Zhang, W. Xu, W. Tong, J. Liao, Y. Zhou, B. Ye and Y. Xie, J. Am. Chem. Soc., 2015, 137, 3102-3108.

10. M. D. Pickett, D. B. Strukov, J. L. Borghetti, J. J. Yang, G. S. Snider, D. R. Stewart and R. S. Williams, J. Appl. Phys., 2009, 106, 074508.

11. Q. Xia, M. D. Pickett, J. J. Yang, M. X. Zhang, J. Borghetti, X. Li, W. Wu, G. Medeiros-Ribeiro and R. S. Williams, Nanotechnology, 2011, 22, 254026.

12. F. M. J Joshua Yang, Matthew D Pickett, and D. R. S. Douglas A A Ohlberg, Chun Ning Lau and R Stanley Williams, Nanotechnology, 2009, 20.

13. B. Qu, A. Younis and D. Chu, Electron. Mater. Lett., 2016, 12, 715-731.

14. M. K. Hota, D. H. Nagaraju, M. N. Hedhili and H. N. Alshareef, Appl. Phys. Lett., 2015, 107, 163106.

15. H. Nili, S. Walia, S. Balendhran, D. B. Strukov, M. Bhaskaran and S. Sriram, Adv. Funct. Mater., 2014, 24, 6741-6750.

16. H. Nili, S. Walia, A. E. Kandjani, R. Ramanathan, P. Gutruf, T. Ahmed, S. Balendhran, V. Bansal, D. B. Strukov, O. Kavehei, M. Bhaskaran and S. Sriram, Adv. Funct. Mater., 2015, 25, 3172-3182.

17. N. Hussein, A. Taimur, W. Sumeet, R. Rajesh, K. Ahmad Esmaielzadeh, R. Sergey, K. Jeeson, K. Omid, B. Vipul, B. Madhu and S. Sharath, Nanotechnology, 2016, 27, 505210.

18. T. Ahmed, S. Walia, J. Kim, H. Nili, R. Ramanathan, E. L. H. Mayes, D. W. M. Lau, O. Kavehei, V. Bansal, M. Bhaskaran and S. Sriram, Nanoscale, 2017, 9, 14690-14702.

19. J.-J. Huang, C.-W. Kuo, W.-C. Chang and T.-H. Hou, Appl. Phys. Lett., 2010, 96, 262901.

20. J. Qi, M. Olmedo, J. G. Zheng and J. Liu, Sci. Rep., 2013, 3, 2405.

21. R. Fahmida, A. Taimur, W. Sumeet, M. Edwin, S. Sharath, B. Madhu and B. Sivacarendran, 2D Mater., 2017, 4, 035008.

22. S. Balendhran, J. Deng, J. Z. Ou, S. Walia, J. Scott, J. Tang, K. L. Wang, M. R. Field, S. Russo, S. Zhuiykov, M. S. Strano, N. Medhekar, S. Sriram, M. Bhaskaran and K. KalantarZadeh, Adv. Mater., 2013, 25, 109-114.

23. Z.-H. Tan, X.-B. Yin and X. Guo, Appl. Phys. Lett., 2015, 106, 023503.

24. D. Lee, D.-j. Seong, I. Jo, F. Xiang, R. Dong, S. Oh and H. Hwang, Appl. Phys. Lett., 2007, 90, 122104.

25. H. K. M Arita, T. Fujii, Y. Takahashi, Thin Solid Films, 2012, 520, 4762-4767.

26. A. A. K. Bessonov, M. N. Petukhov, D. I. Allen, M. Ryhänen, T. Bailey, M. J. A., Nat. Mater., 2015, 14, 199-204.

27. M. A. Camacho-López, L. Escobar-Alarcón, M. Picquart, R. Arroyo, G. Córdoba and E. Haro-Poniatowski, Opt. Mater., 2011, 33, 480-484.

28. D. V. Pham, R. A. Patil, J.-H. Lin, C.-C. Lai, Y. Liou and Y.-R. Ma, Nanoscale, 2016, 8, 5559-5566. 
29. P. Spevack and N. Mclntyre, J. Phys. Chem., 1992, 96, 9029-9035.

30. X. K. Hu, Y. T. Qian, Z. T. Song, J. R. Huang, R. Cao and J. Q. Xiao, Chem. Mater., 2008, 20, 1527-1533.

31. P. A. Spevack and N. S. McIntyre, J. Phys. Chem., 1993, 97 11020-11030.

32. C. Wang, I. Irfan, X. Liu and Y. Gao, J. Vac. Sci. Technol. B, 2014, 32, 040801.

33. D. O. Scanlon, G. W. Watson, D. J. Payne, G. R. Atkinson, R. G. Egdell and D. S. L. Law, J. Phys. Chem. C, 2010, 114, 4636-4645.

34. J. G. Choi and L. T. Thompson, Appl. Surf. Sci., 1996, 93, 143-149.

35. J. Baltrusaitis, B. Mendoza-Sanchez, V. Fernandez, R. Veenstra, N. Dukstiene, A. Roberts and N. Fairley, Appl. Surf. Sci., 2015, 326, 151-161.

36. S. S. Sunu, E. Prabhu, V. Jayaraman, K. I. Gnanasekar, T. K. Seshagiri and T. Gnanasekaran, Sens. Actuators, B, 2004, 101, 161-174.

37. S. Hao, B. Yang and Y. Gao, Phys. Status Solidi B, 2017, 254, 1600245.

38. L. Kumari, Y.-R. Ma, C.-C. Tsai, Y.-W. Lin, S. Y. Wu, K.-W. Cheng and Y. Liou, Nanotechnology, 2007, 18, 115717.

39. D. Ielmini, Semicond. Sci. Technol., 2016, 31, 063002.

40. W. Zongwei, K. Jian, Y. Zhizhen, F. Yichen, L. Yaotian, C. Yimao, H. Ru and W. Yangyuan, Nanotechnology, 2017, 28, 055204.

41. J.-Y. Chen, C.-W. Huang, C.-H. Chiu, Y.-T. Huang and W.-W. Wu, Adv. Mater., 2015, 27, 5028-5033.

42. H. Efeoglu, S. Güllülü and T. Karacali, Appl. Surf. Sci., 2015, 350, 10-13.

43. E. Lim and R. Ismail, Electronics, 2015, 4, 586.

44. G. Zhou, S. Duan, P. Li, B. Sun, B. Wu, Y. Yao, X. Yang, J. Han, J. Wu, G. Wang, L. Liao, C. Lin, W. Hu, C. Xu, D. Liu, T. Chen, L. Chen, A. Zhou and Q. Song, Adv. Electron. Mater., 2018, DOI: 10.1002/aelm.201700567.

45. S.-Y. Wang, C.-W. Huang, D.-Y. Lee, T.-Y. Tseng and T.-C. Chang, J. Appl. Phys., 2010, 108, 114110.

46. Z. Xu, L. Yu, Y. Wu, C. Dong, N. Deng, X. Xu, J. Miao and Y. Jiang, Sci. Rep., 2015, 5, 10409.

47. I. Valov, E. Linn, S. Tappertzhofen, S. Schmelzer, J. van den Hurk, F. Lentz and R. Waser, Nat. Commun., 2013, 4, 1771.

48. S. Gao, C. Song, C. Chen, F. Zeng and F. Pan, J. Phys. Chem. C, 2012, 116, 17955-17959.

49. J. W. Rabalais, R. J. Colton and A. M. Guzman, Chem. Phys. Lett., 1974, 29, 131-133.

50. S. Menzel, U. Böttger, M. Wimmer and M. Salinga, Adv. Funct. Mater., 2015, 25, 6306-6325. 